find that it is in radium-actinium, actinium emanation, and the three C-products mentioned above that relatively strong slow groups are present, while for other elements the intensities of these groups are practically on the limit of observation.

From the relative intensities of different 'fine-structure' groups of a given nucleus, it is possible also to determine the corresponding $j$ 's. For thorium $\mathrm{C}$, for example, the calculated (for $j=0$ ) and observed intensities of different $\alpha$-groups are shown in the accompanying table :

\begin{tabular}{|c|ccccc|}
\hline $\begin{array}{c}\text { Name of the } \\
\text { group }\end{array}$ & $a_{1}$ & $\alpha_{0}$ & $a_{2}$ & $a_{4}$ & $\alpha_{3}$ \\
\hline $\begin{array}{c}\text { Calculated in- } \\
\text { tensity (for } j=0 \text { ) }\end{array}$ & 1.0 & 0.7 & 0.03 & 0.005 & 0.004 \\
\hline $\begin{array}{c}\text { Ob b e r v e d } \\
\text { intensity } . \cdots\end{array}$ & 0.3 & 1.0 & 0.03 & 0.002 & 0.02 \\
\hline $\begin{array}{c}\text { Relative de- } \\
\text { crease }\end{array}$ & 17 & 3.5 & 5.0 & 13 & 1 \\
\hline
\end{tabular}

It may be concluded that for $\alpha_{1}$ and $\alpha_{4}$ we have $j=3$, for $\alpha_{0}$ and $\alpha_{2}, j=2$ and for $\alpha_{3}, j=0$ or 1 . If we assume the spin-difference between the normal state of the thorium $\mathrm{C}$ and thorium $\mathrm{C}^{\prime \prime}$ nuclei to be 3 , we arrive at the following system of excited levels of thorium $\mathrm{C}^{\prime \prime}$ : $\alpha_{1}(S) ; \alpha_{0}(p) ; \alpha_{2}(p) ; \alpha_{4}(S) ; \alpha_{3}(d$ or $f)$.

It is not possible at the moment to compare the energy levels for all nuclei for which 'fine-structure' is found, as the experimental evidence is not yet complete. The striking similarity between the levels of the nuclei of thorium $\mathrm{C}^{\prime \prime}$ and actinium $\mathrm{X}$ is noteworthy: these two level systems differ from one another only by 40 per cent, which is evidently connected with the change of radius by 20 per cent $\left(6.6 \times 10^{-13}\right.$ and $\left.7.9 \times 10^{-13}\right)$.

If we compare, however, the levels excited by $\alpha$-decay with the level system of the radium $\mathbf{C}^{\prime}$ nucleus $^{4}$ excited by $\beta$-decay, we find at once that the energy differences are in the last case about four times larger, which supports the hypothesis that $\gamma$-radiation by $\beta$-decay is due to proton transitions ${ }^{5}$.

Physico-Mathematical Institute,

$$
\text { G. Gamow. }
$$

Academy of Sciences of U.S.S.R., Leningrad. March 17.

\footnotetext{
1 Gamow, Nature, 126, 397, Sept. 13, 1930.

Gamow, Nature, 129, 470, March 26, 1932

${ }^{3}$ Rosenblum, "Str. fine des rayons alpha". Paris, 1932

Gamow, NATURE, 131, 433, March 25, 1933.

5 Gamow, NATURE, 131, 57, Jan. 14, 1933.
}

\section{Periodicity in the Solarisation of Calcite}

REPEATED solarisation due to a prolonged exposure to the light of a quartz mercury lamp was observed by the photoluminescence exhibited by calcite from Nabeto. A definite quantity $(0 \cdot 045 \mathrm{gm}$.$) of$ the finely granulated specimen was put at suitable intervals of time into the depressions of the sample holder ${ }^{1}$ placed $15 \mathrm{~cm}$. underneath the lighted lamp (100 volts, $3 \cdot 1$ amp.) which was employed as a constant source of exciting light. Thus a set of samples was subjected to excitation. A panchromatic plate was then applied, immediately after cessation of excitation, on the sample holder, thus charged with a set of the samples excited for discrete durations of time, the distance between the surface of samples and the photographic plate being $3 \mathrm{~mm}$. After being left two hours, the plate was developed, and the photographic densities of the images generated on the plate by the action of phosphorescent light of the excited samples were determined by means of a microphotometer. The results are shown graphi cally in Fig. 1, which gives the growth curve of photoluminescence, indicating its periodic reversal under the exciting rays. The ordinates represent the density corresponding to the intensity of phosphorescence and the abscissæ the duration of excitation.

As will be seen, the first maximum of the intensity of luminescence appears at about two hours irradiation. With a still longer irradiation, the luminescence gradually loses in intensity owing to the solarisation of the sample. If the excitation is continued further, a minimum of the intensity of luminescence is reached at about twenty-five hours exposure, and a gradual increase of intensity is then observed again up to the second maximum attained at about thirty-three

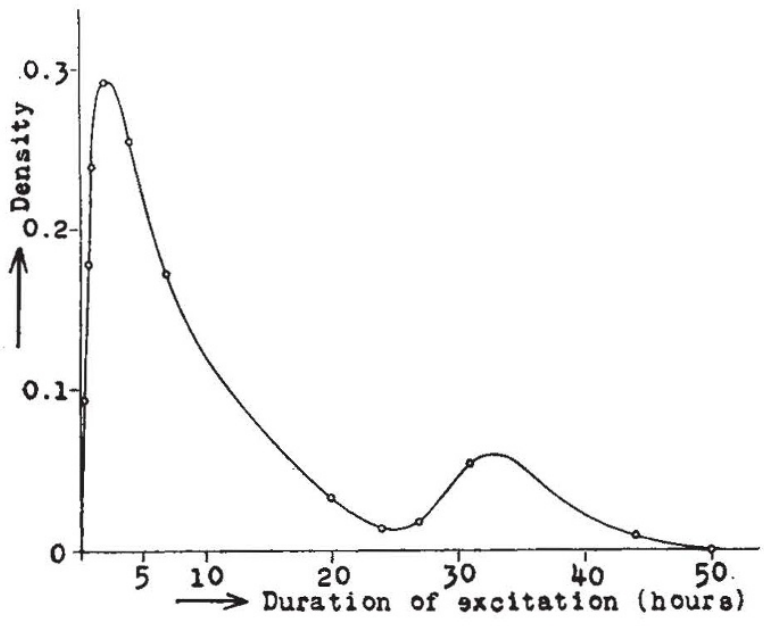

FIG. 1.

hours irradiation, after which another reversal begins again. Of course, the intensity of photoluminescence corresponding to the second maximum is very weak as compared with that corresponding to the first maximum. The result is quite reproducible with new samples.

Hence it can be concluded that the phenomenon of solarisation of calcite, by which is meant the reversal of its photoluminescence process under the continual action of the light of quartz mercury lamp, is apparently analogous in character to the wellknown photographic reversal.

S. IIMORr.

Institute of Physical and Chemical Research, Tokyo, Japan. Feb. 2.

${ }^{1} \mathrm{~S}$. Yimori and E. Iwase, Sci. Pap. Instit. Phys. Chem. Res., 16, 41 1931.

\section{Origin of Cosmic Radiation}

EXPERIMENTS seem to show that the cosmic rays are very fast particles, coming from world space; but at present there is no acceptable theory of the phenomena giving rise to them. Neither Millikan's nor Jeans's hypotheses of nuclear integration or annihilation processes, nor Regener's ${ }^{1}$ or Swann's ${ }^{2}$ theories, seem to be in accordance with the latest experimental results. 